\title{
Contribuições para 0 conhecimento da fauna helmintolojica brazileira
}

\author{
pelo \\ DIR. LAURO TRAVASSOS.
}

III

Sobre as especies brazileiras do genero Tetrameres CREPLIN, 1846.

(Com as estampas 16-23).

\section{Beitraege zur Kenntniss der brasilianischen Helmintheni̊auna von}

\section{DR. LAURO TRAVASSOS}

III

Ueber die brasilianischen Arten des Genus Tetrameres CREPLIN, 1846. (Mit Tafel. 16-23).

Tetrameres CREPLIN, 1846.

Sin. Tropisurus DIESING, 1835, nom. praeocc.

Tropidurus WIEGMANN, 1835, nom. praeocc.

Tetrameres CREPLIN, 1846.

Tropidocerca DIESING, 1850.

Astomum SCHLOTTHAUBER, 1859.

Acanthophorus v. LINSTOW, 1879.

Este genero se caraterisa desde logo, pelo grande dimorfismo sexual, o que obriga a descrição especial para cada sexo.

Femea: O corpo é arredondado, de côr vermelha, com estriações transversaes profundas, sobre tudo na parte central onde são
Tetrameres CREPLIM, 1846.

Syn. Tropisurus DIESING, 1835, nom. praeocc.

Tropidurus WIEGMANN, 1835, nom. praeocc.

Tetrameres CREPLIN, 1846.

Tropidocerca DIESING, 1850.

Astomum SCHLOTTHAUBER,1859.

Acanthophorus v. LINSTOW, 1879.

Diese Gattung ist sofort an dem ausgepraegten sexuellen Dimorphismus kenntlich, welcher eine getrennte Beschreibung der Geschlechter bedingt.

Weibchen: Koerper rundlich, rot, mit tiefen transversalen Furchen, besonders im 
mais afastadas, e 4 depressões lonjitudinaes ao longo dos campos medianos e lateraes, que dá ao parasito aspeto canelado; as vezes o corpo sofre uma torsão no sentido do eixo lonjitudinal; as extremidades se apresentam como dois apendices conicos.

O tubo dijestivo é constituido pelas seguintes partes: abertura bucal, guarnecida de tres labios pequenos e seguida de uma capsula bucal quitinosa, farinje e esofago musculosos, intestino constituido por um grande saco de paredes delgadas, geralmente repleto de detritos negros e terminando no poro anal por um tubo muito fino.

O aparelho genital é muito desenvolvido; o utero, que contem um numero elevadissimo de ovos em diversos estadios de evolução, ocupa grande parte da cavidade geral; os ovos elipsoides, de casca delgada, contem no momento da postura um embrião completamente desenvolvido; a vulva fica situada na extremidade posterior, pouco acima do anus.

Macho; o corpo é filiforme, de côr branca, geralmente guarnecido de espinhos ao longo dos campos medianos e lateraes, com estriações transversaes mais ou menos acentuadas. $\mathrm{O}$ aparelho dijestivo é constituido, como na femea, por abertura bucal guarnecida de tres labios pequenos, por capsula bucal quitinosa, farinje e esofago musculosos e pelo intestino; este, porem, não é saciforme nem contem detritos negros. O aparelho genital é muito desenvolvido; ha dois espiculos, um dos quaes, muito grande, chega a medir $2 / 3$ do comprimento do parasito. Nem sempre ha papilas na extremidade posterior.

Especie tipo: Tetrameres paradoxa (DIESING, 1835).

Habitat: Proventriculo de aves e reptis; as femeas se alojam nas glandulas de LIEBERKUEHN, produzindo a atrofia do epitelio glandular, e os machos ficam na cavidade do orgão.

A presença destes parasitos é denunciada, desde logo, pelas femeas visiveis como manchas vermelhas atravez da parede externa do proventriculo. mittleren Teile, wo sie mehr entfernt stehen, und vier laenglichen Depressionen, entsprechend den Median-und Seitenlinien, welche den Parasiten kanneliert erscheinen lassen; manchmal findet eine Torsion in der Laengsachse statt; die beiden Enden des Koerpers bilden konische Anhaengsel.

- Der Digestionstrakt besteht aus den folgenden Teilen: Mundoeffnung mit drei kleinen Lippen, gefolgt von einer chitinoesen Mundkapsel, muskuloesem Pharynx, ebensolchem Oesophagus, und duennwandigem, weitem sackfoermigem Darme, der gewoehlich mit Detritus gefuellt ist und durch ein feines Rohr in den Porus analis muendet.

Der Genitalapparat ist sehr entwickelt; der Uterus enthaelt eine sehr grosse Zahl von Eiern in verschiedenen Entwicklungsstadien und nimmt einen grossen Teil der Koerperhoehlung ein; die duennschaligen, eifoermigen Eier enthalten bei der Ablage einen voellig entwickelten Embryo; die Vulva befindet sich am Hinterende in der Naehe des Anus.

Maennchen: Koerper fadenfoermig, weiss, gewoebnlich mit Dornen laengs der Medianund Seitenlinien und mit mehr oder weniger ausgesprochenen queren Furchen. Der Darmkanal, wie bei Weibchen aus folgenden Teilen bestehend: Mund mit drei kleinen Lippen, chitinoese Mundkapsel, Pharynx und Oesophagus beide deutlich muskuloes, endlich der Darm, der weder sackfoermig ist, noch einen schwarzen Inhalt zeigt. Der Geschlechtsapparat ist sehr entwickelt; es finden sich zwei Spicula, von denen das eine sehr gross ist und $2 / 3$ der Laenge des Wurmes erreicht. Das Hinterende manchmal mit $\mathrm{Pa}$ pillen.

Typische Art: Tetrameres paradoxa (DIESING, 1835).

Fundort: Proventriculus von Voegeln und Reptilien. Die Weibchen befinden sich in den LIEBERKUEHN Druesen, deren Epithel atrophiert; die Maennchen finden sich frei in der Hoelung des Organes.

Die Weibchen sind schon durch die aeussere Wand des Proventriculus in Form 
Os autores tem incluido este genero na familia Filariidae, porem este grupo de parasitos se afasta de modo tão notavel dos de mais nematoideos, que nos parece razoavel crear-se para ele uma familia a parte "Tetrameridae", tendo como carateres os do genero.

O grande dimorfismo sexual destes nematoideos ocasionou muitos enganos aos helmintolojistas que com eles se ocuparam; assim é, que alguns autores classificaram os dois sexos como especies, e mesmo generos diversos, com isto complicando extremamente o assunto.

Segue uma lista das especies deste genero com as respetivas sinonimias e hospedadores.

\section{1). Tetrameres paradoxa (DIESING, 1835)}

Sin. Tropisurus paradoxus DIESING, 1835, nec v. LINSTOW, 1877.

Tropisurus paradoxus DUJARDIN, 1845.

Tropidocerca paradoxa DIESING, 1851

Tropidocerca paradoxa v. LINSTOW, 1879.

Tropidocerca paradoxa DRASCHE, 1883.

Hosp. Catharistes atratus BARTRAM, 1791.

Sirnium perspicillatum (LATH. 1781).

Falco communis GM., 1788.

Scolopax rusticola L. 1766.

Grus grus (L., 1766).

Anthropoides virgo (L., 1766).

Mergus albellus L., 1766.

Pellidina alpina (L., 1766).

Anser ferus SCHAEFF., 1889.

Numenius arquatus (L., 1766).

\section{2). Tetrameres gynaecophila (MOLIN, 1858).}

Sin. Tropidocerca gynaecophila MOLIN, 1858.

Tropidocerca gynaecophila v. LINSTOW, 1879. roter Flecken erkennbar, wodurch sich die Gegenwart der Parasiten sofort verraet.

Die Autoren haben dieses Genus zu den Filariidae gestellt, doch entfernt sich diese Gruppe von Parasiten so sehr von den anderen Nematoden, dass es mir angebracht erscheint, fuer diesselbe eine separate Familie Tetrameridae mit den Charakteren der Gattung zu errichten.

Der ausgesprochene Geschlechtsdimorphismus dieser Nematoden hat die Helminthologen zu vielen Irrtuemern verfuelirt, so dass einige der Autoren, die sich mit ihnen befassten, die beiden Geschlechter als verschiedene Arten oder sogar Gattungen auffassten, wodurch die Frage ausserordentlich kompliziert wurde.

Ich lasse ven den Arten dieser Gattung eine Liste folgen, mit Angabe der Synonymie und der Wirtstiere.

1). Tetrameres paradoxa (DIESING, 1835).

Syn. Tropisurus paradoxus, DIESING, 1835, nec. v. LINSTOW, 1877.

Tropisurus paradoxus DUJARDIN, 1845.

Tropidocerca paradoxa DIESING, 1851.

Tropidocerca paiadoxa v. LINSTOW, 1879.

Tronidocerca paradoxa DRASCHE, 1883.

Wirte: Catharistes atratus BARTRAM, 1791.

Syrnium perspicillatum (LATH.1781)

Falco communis GM., 1788.

Scolopax rusticola L. 1766.

Grus grus (L., 1766).

Anthropoides virgo (L. 1766).

Mergus albellus L. 1766.

Pelidna alpina (L. 1766).

Anser ferus SCHAEFF., 1789.

Numenius arquatus (L., 1766).

2). Tetrameres gynaecophila IMOLIN, 1858).

Syn.: Tropidocerca gynaecophila MOLIN, 1858.

Tropidocerca gynaecophila v. LINSTOW, 1879. 
Hosp. Nycticorax nycticorax (L., 1766).

3). Tetrameres inflata (DIESING, 1860).

Sin. Tropidocerca inflata DIESING, 1860. Tropidocerca paradoxa v. LINSTOW, 1877, nec DIESING, 1835.

Tropidocerca inflata v. LINSTOW, 1879.

Hosp. Numenius arquatus (L., 1766).

Charadrius pluvialis L., 1766.

Ciconia nigra (L., 1766).

Anas boscas L., 1766.

Harelda glacialis (L., 1766).

Somateria molissima (L., 1766).

Mergus albellus L., 1766.

Merganer serratus (L., 1766).

4). Tetrameres unispina (DIESING, 1860).

Sin. Tropidocerca unispina DIESING, 1860.

Tropidocerca unispina v. LINSTOW, 1879.

Hosp. Corono cornix (L., 1766).

5). Tetrameres bispinosa (MOLIN, $1860)$.

Sin. Tropidocerca bispinosa MOLIN, 1860.

Tropidocerca bispinosa v. LINSTOW, 1879.

Hosp. Scincus officinalis (LAUR., 1767.)

6). Tetrameres fissispina (DIESING, 1860).

Sin. Tropidocerca fissispina DIESING, 1860.

Acanthophorus tenuis v. LINSTOW, 1876.

Acanthophorus horridus v. LINSTOW, 1876.

Tropidocerca fissispina v. LINSTOW, 1879.

Tropidocerca fissispina v. LINSTOW, 1899.

Tropidocerca inflata ZUERN 1882, nec DIESING, 1860.

Tropisorus fissispinus NEUMANN, 1888.
Wirt: Nycticorax nycticorax (L., 1766).

3). Tetrameres inflata (DIESING, 1860).

Syn. Tropidocerca inflata DIESING, 1860.

Tropidocerca paradoxa $\mathrm{v}$. LINSTOW, 1877 nec DIESING, 1835.

Tropidocerca inflata v. LINSTOW, 1879.

Wirte: Numenius arquatus (L., 1766).

Charadrius pluvialis L., 1766.

Ciconia nigra (L., 1766).

Anas boscas L., 1766.

Harelda glacialis (L. 1766).

Somateria mollissima (L., 1766).

Mergus albellus L., 1766.

Merganser serratus (L., 1766).

4). Tetrameres unispina (DIESING, 1860).

Syn.: Tropidocerca unispina DIESING, 1860.

Tropidocerca unispina v. LINSTOW, 1879.

Wirt: Corono cornix (L., 1766).

5). Tetrameres bispinosa (MOLIN, 1860).

Syn.: Tropidocerca bispinosa MOLIN, 1860.

Tropidocerca bispinosa v. LINSTOW, 1879.

Wirt: Scincus officinalis (LAUR. 1766).

6). Tetrameres fissispina (DIESING, 1860).

Syn.: Tropidocerca fissispina DIESING, 1860.

Acanthophorus tenuis v. LINSTOW, 1876.

Acanthophorus horridus v. LINSTOW, 1876.

Tropidocerca fissispina v. LINSTOW, 1879.

Tropidocerca fissispina v. LINSTOW, 1899.

Tropidocerca inflata ZUERN 1882, nec DIESING, 1860.

Tropisurus fissipinus NEUMANN, 1888. 
Tropisurus fissispinus RAILLIET, 1895.

Hosp. Fulica atra L., 1766.

Anas boscas L. , 1766

Gallus domesticos L., 1766.

7). Tetrameres globosa. (v. LINSTOW, 1879).

Sin. Tropidocerca globosa v. LINSTOW, 1879.

Hosp. Fulica atra L., 1766.

8). Tetrameres inermes ( $v$. LINSTOW, 1879).

Sin. Tronidocerca inermis v. LINSTOW, 1879.

Hosp. Astur palumbarius L., 1766.

9). Tetrameres certa (LEIDY, 1886).

Sin. Tropidocerca certa LEIDY, 1886.

Hosp. Diomedea exulans L., 1766.

10). Tetrameres contorta (WIEDMANN, 1913.)

Sin. Tropidocerca contorta WIEDMANN, 1913.

Hosp. Dichocerus bicornis (L., 1766).

11). Tetrameres nouveli (SEURAT, 1914).

Sin. Tropidocerca nouveli SEURAT, 1914. Hosp. Himantopus himantopus (L., 1766)

12) Tetrameres coccinea (SEURAT, 1914).

Sin. Tropidocerca coccinea SEURAT, 1914,

Hab. Phoenicopterus roseus PALL., 1811.

13). Tetrameres cruzi. n. sp.

Hosp. Bucco swainsoni GRAY, 1847. Melanerpes flavifrons (VIEILL., 1818)

14). Tetrameres minima n. sp.

Hosp. Tachyphonus cristatus bruneus (SPIX, 1824).

V. LINSTOW, no Compendium der Helminthologie, assinala o $T$. paradoxa como parasito de aves, de grupo e destribuição geografica muito diversa daquela $\epsilon \mathrm{m}$ que foram encontrados os exemplares que serviram
Tropisuras fissispinus RAILLIET, 1895.

Wirte: Fulica atra L., 1766.

Anas boscas L., 1760 .

Gallus domesticus L., 1766.

7). Tetrameres glabosa (v. LINSTOW, 1879),

Syn.: Tropidocerca globosa v. LINSTOW, 1879.

Wirt: Fulica atra L., 1766.

8). Tetrameres inermes (v. LINSTOW, 1879).

Syn.: Tropidocerca inermis v. LINSTOW, 1879.

Wirt: Astur palumbarius L., 1766.

9). Tetrameres certa (LEIDY, 1886).

Syn. Tropidocerca certa LEIDY, 1886.

Wirt: Diomedea exulans L., 1766.

10). Tetrameres contorta (WIEDMANN, 1913).

Syn.: Tropidocerca contorta WIELMANN, 1913.

Wirt: Dichocerus bicornis (L., 1766).

11) Tetrameres nouveli (SEURAT, 1914).

Syn. : Tropidocerca nouveii SEURAT, 1914.

Wirt: Himantopus himantopus (L., 1766).

12) Tetrameres coccinea (SEURAT, 1914).

Syn.: Tropidorcerca coccinea SEURAT, 1914.

Wirt: Phoenicopterus roseus PALL., 1811.

13) Tetrameres cruzi n. sp.

Wirte: Bucco swainsonı GRAY, 1847.

Melanerpes flavifrons (WIEILL, 1818).

14) Tetrameres minima n. sp.

Wirt: Tachyphonus cristatus bruneus (SPIX, 1824). 
para a descrição de DIESING, parecendo ter havido confusão com os $T$. inflata e fissispina.

Da lista das especies acima são encontradas no Brazil as seguintes:

1). Tetrameres paradoxa (DIESING, 1835).

2). Tetrameres fissispina (DIESING, 1860).

3). Tetrameres cruzi n. sp.

4). Tetrameres minima n. $\mathrm{sp}$.

\section{Tetrameres paradoxa (DIESING, 1835).} (Est 18, Fig. 5)

Não conseguimos obter exemplares deste parasito, embora esaminassemos numero bastante elevado de Catharistes atrata, provenientes de diversas localidades do Estado do Rio de Janeiro.

Reproduzimos a descrição orijinal de DIESING e a de DRASCHE, bem comio as figuras deste ultimo.

"Corpus maris subcylindricum, gracile; feminae subglobosum fasciis 4 longitudinalibus oppositis aequidistantibus signatum. Caput breve conicum corpore continuum. Os terminale orbiculare. Extremitas caudalis maris recta acuta subtus excavata, pene filiforme in vagina tubulosa ; feminae conica brevis, apertura genitali antrorsum sita.-Corpus maris antrorsum magis attenuatum, arcuatum album; feminae subglobosum utrinque acute conicum, zonis transversis numerosissimis, sanguineo-rubum. Long. mar. 5-6"', crassit. $1 / 3^{\prime \prime \prime}$; fem. 3-4"', crassit. 3"',." E' este o resumo da descrição de DRASCHE:

Boca redonda, sem labios, com 4 papilas; capsula bucal curta; esofago constituido por duas partes, uma anterior delgada e outra posterior larga; intestino amplo, separado do reto por um estrangulamento; orgãos sexuaes masculinos começando ao nivel da extremidade posterior do esofago; vaso deferente com uma dilatação piriforme na extremidade posterior; duas espiculas desiguais, a direita mede $0,48 \mathrm{~mm}$. e a esquerda $3 \mathrm{~mm}$. e mais.
In seinem Compendium der Helminthologie verzeichnet LINSTOW $T$. paradoxus als Parasit von Voegeln, die nach Stellung und geographischer Verbreitung sehr von denjenigen abweichen, in welchen die der DIESINGschen Beschreibung zu Grunde liegenden gefunden wurden; es scheint eine Verwechslung mit $T$. inflata und fissispina stattgefunden $\mathrm{zu}$ haben.

Aus der oben gegebenen Liste wurden in Brasilien folgende Arten gefunden:

1) Tetrameres paradoxa (DIESING, 1835).

2) Tetrameres fissispina (DIESING, 1860).

3) Tetrameres cruzi n. sp.

4) Tetrameres minima n. sp.

\section{Tetrameres paradoxa (DIESING, 1835)}

(Taf. 18, Fig. 5 )

Obwohl ich ziemlich viele Catharistes atrata aus verschiedenen Gegenden des Staates Rio de Janeiro untersuchte, fand ich keine Exemplare dieses Wurmes. Ich gebe dis: Originalbeschreibungen von DIESING und DRASCHE und die Figuren des letzteren wieder.

"Corpus maris subcylindricum, gracile; feminae subglobosum, fasciis quatuor longitudinalibus oppositis aequedistantibus signatum. Caput breve conicum corpore continuum. Os terminale orbiculare. Extremitas caudalis maris recta acuta subtus excavata, pene filiformi in vagina tubulosa; feminae conica brevis, apertura genitali antrorsum sita.-Corpus maris antrorsum magis attenuatum, arcuatum album; feminae subglobosum utrinque acute conicum, zonis transversis numerosimis, sanguineo-rubrum. Long. mar 56"', crassit. 1/3"', fem. 3-4"', crassit. 3"'. "

Es folgt ein Auszug der Beschreibung von DRASCHE:

Mund rund, ohne Lippen, mit vier $\mathrm{Pa}$ pillen; Mundkapsel kurz; Oesophagus aus zwei Teilen bestehend, von denen der vordere eng, der hintere weit ist; Darm weit, vom Rektum durch eine Einschnuerung getrennt; aehnliche Geschlechtsorgane auf der Hoehe des hinteren Oesophagusendes beginnend; vas deferens am Hinterende mit 
Tetrameres fissispina (DIESING, 1835).

(Est. 16 ; est. 17 , fig. 2 ; est. 18 , fig. $6-7$; est. 19 , fig. $9-10$; est. $20-21$; ).

Femea: Comprimento: 3 a $5 \mathrm{~mm}$.

Largura: 2 a $3 \mathrm{~mm}$.

Corpo subglobular, de cor vermelha, com fortes estriaçōes transversaes e quatro depressões lonjitudinaes, que correspondem aos campos medianos e lateraes. As extremidades, cefalica e caudal, se apresentam como prolongamentos delgados, de forma conica, retrateis e muito moveis sobretudo a primeira. A extremidade anterior, quando distendida, mede cerca de $1 \mathrm{~mm}$. e a posterior $0,9 \mathrm{~mm}$.

O tubo dijestivo (Fig. 1 ) é constituido pelas seguintes partes: capsula bucal, farinje, esofago e intestino. A capsula bucal em forma de tonel, mede cerca de $0,020 \mathrm{~mm}$. de comprimento por 0,014 de maior largura; o farinje muito longo, mede $0,4 \mathrm{~mm}$ de comprimento por $0,03 \mathrm{~mm}$. de largura, é constituido por grandes celulas não estratificadas e a cavidade é de seção triangular; esofago, que descreve sinuosidades, mede $2 \mathrm{~mm}$. de comprimento por $0,18 \mathrm{~mm}$. de largura maxima; é constituido por celulas menores que as do farinje e dispostas em camadas estratificadas; a cavidade é de seção circular; o intestino é um grande saco, geralmente contendo detritos negros e por isso facilmente visivel a olho $\mathrm{nu}$, piriforme, sendo a extremidade posterior muito fina; mede, quando comprimido entre lamina e laminula, mais ou menos 3 mm. de comprimento por $2,5 \mathrm{~mm}$. de maior largura; é constituido por uma delgada camada de celulas não estratificadas, exceto em torno da abertura do esofago, onde se encontram grandes celulas glandulares piriformes (Est, 21, fig. 14); o anus fica situado a 0,25 mm. da extremidade posterior e é precedido de um reto longo e de paredes espessas.

Os orgãos genitaes são muito desenvolvidos e ocupam grande parte da cavidade do nematoideo; o utero duplo se apresenta em forma de tubos, de grossura irregular, muito longos, formando numerosas alças, repletas de ovos em diversos estadios de evolução; são constituidos por paredes tenues e aprenta receptaculos seminaes bem desenvolvidos; birnfoermiger Erweiterung; zwei ungleiche Spicula, das rechte $0.48 \mathrm{Mm}$. lang, das linke $3 \mathrm{Mm}$. oder mehr.

Tetrameres fissispina (DIESING, 1835).

(Taf 16; Taf. 17, Fig. 2; Taf. 18, Fig. 6-7; Taf. 16, Fig. 9-10; Taf. 20-21).

Weibchen: Laenge: 3-5 Mm.

Breite: 2-3 Mm.

Koerper rundlich, rot, stark quergestreift, mit vier Laengsfurchen, die den Mittel-und Seitenlinien entsprechen. Kopf - und Schwanzende erscheinen als feine konische Fortsaetze; sie sind retraktil und sehr beweglich, besonders das erstere. Das Vorderende misst ausgestreckt za. 1 Mm., das Hinterende $0,9 \mathrm{Mm}$.

Der Digestionstractus (Fig. 1.) besteht aus folgenden Teilen: Mundkapsel, Pharynx, Oesophagus und Darm. Die tonnenfoermige Mundkapsel ist $0,020 \mathrm{Mm}$. lang bei groesster Breite von 0,014 ; Pharynx sehr lang, 0,4 Mm. zu 0,03 Breite, aus grossen nicht geschichteten Zellen gebildet, die Hoehlung auf dem Durchschnitt dreieckig; der Oesophagus ist gewunden, $2 \mathrm{Mm}$. lang bei einer groessten Breite von $0,18 \mathrm{Mm}$. und aus Zellen gebildet, welche kleiner als diejenigen des Pharynx und mebrere Schichten bilden, seine Hoehlung ist im Querschnitte rund; Darm in Form eines grossen Sackes, gewoehnlich mit schwarzem Detritus und daher leicht mit blossem Auge zu erkennen, birnfoermig mit sehr duennem hinterem Ende, misst vom Deckglase komprimiert za. $3 \mathrm{Mm}$. Laenge bei 2,5 groesster Breite; er besteht aus einer duennen Lage nicht geschichteter Zellen, ausser um die Oesophagusmuendung, wo er grosse birnfoermige Druesenzellen zeigt (Taf. 21, Fig. 14), der Anus liegt 0,25 Mm. vom Hinterende am Ende des langen und dickwandigen Rectums.

Die Genitalorgane sind stark entwickelt und nehmen einen grossen Teil der Koerperhoehle ein, der doppeite Uterus erscheint in Form von langen, zahlreiche Schlingen bildenden Roehren von wechselnder Dicke, die von Eiern in verschiedenen Entwicklungsstadien erfuellt sind; sie sind duennwandig und zeigen wohl entwickelte Receptacula seminis; die Ovarien erscheineli als lange Roehren, die in der Mitte am Dicksten sind 
os ovarios são representados por longos tubos mais largos na parte central e formados por grandes celulas de protoplasma granulado; a vulva, situada na extremidade posterior, logo acima do anus é precedida de uma vajina longa a qual apresenta, logo no inicio, um diveiticulo que termina em uma dilatação em forma de ampola que nos exemplares adultos se acha repleta de ovos: estes são elipsoides, medem $0,043 \mathrm{~mm}$. de comprimento por $0,04 \mathrm{~mm}$. de largura maxima; apresentando na ocasião da postura um embrião completamente desenvolvido. O póro excretor fica situado a cerca de $0,15 \mathrm{~mm}$. da extremidade anterior, mais ou menos ao nivel dos ganglios nervosos que formam a coleira esofajiana.

Macho: Comprimento 4 a $5 \mathrm{~mm}$.

Largura $0,16 \mathrm{~mm}$.

Corpo filiforme de cor branca, apresentando, ao longo das linhas medianas, e lateiaes espinhos dirijidos para traz e afastados de cerca de $0,10 \mathrm{~mm}$. na parte media do corpo e de $0,02 \mathrm{~mm}$. na extremidade posterior. A extremidade caudal termina em uma ponta em forma de aculeo de $0,004 \mathrm{~mm}$. de comprimento.

O tubo dijestivo (Est. 18, fig. 7) é bem diferente do da femea; a capsula bucal é muito pequena e cilindrica, mede mais ou menos $0,024 \mathrm{~mm}$. de comprimento por $0,008 \mathrm{~mm}$. de largura: o farinje mede cerca de $0,25 \mathrm{~mm}$. de comprimento por $0,012 \mathrm{~mm}$. de largura; o esofago mede $0,75 \mathrm{~mm}$. de comprimento por $0,055 \mathrm{~mm}$. de largura maxima; o intestino é um tubo mais ou menos da mesma largura do esofago, na parte anterior, tornandose muito fino na posterior; o anus fica situado a $0,07 \mathrm{~mm}$. da extremidade posterior.

Os testiculos são muito desenvolvidos e são providos de um canal deferente que apresenta grande dilatação na extremidade anterior; os espiculos medem 0,068 e $0,291 \mathrm{~mm}$. de comprimento. Abaixo do anus existem 8 espinlos de cada lado, dos quaes 3 nos campos lateraes e 5 na face ventral, proximos do campo mediano. und aus grossen Zellen mit koernigem Protoplasma bestehen; auf eine lange Vagina folgt die Vulva, welche an Hinterende dicht ueber den Anus liegt; erstere zeigt gleich anfangs ein Diverticulum, welches in einer ampullenfoermigen Erweiterung endigt, welche bei erwachsenen Individuen mit Eiern gefuellt erscheint. Die Eier sind ovoid, 0,043 Mm. lang und 0,024 breit und enthalten zur Zeit der Ablage einen vollstaendig entwickelten Embryo. Der Porus excretoritus liegt 0,15 $\mathrm{Mm}$. vom Vorderende, ungefaehr auf der Hoehe der Ganglien, welche den Schlundring bilden.

Maennchen: Laenge 4-5 Mm.

Breite 0,15 $\mathrm{Mm}$.

Koerper fadenfoermig, weiss, laengs der Mittel-und Seitenlinien mit rueckwaerts gerichteten Stacheln in Abstaenden von 0,10 $\mathrm{Mm}$. in der Koerpermitte und 0,02 am Schwanzende; dieses endet in eine $0,004 \mathrm{Mm}$ lange stachelfoermige Spitze.

Der Tractus digestivus (Taf. 18, Fig. 7 ) ist von demjenigen des Weibchens sehr verschieden; die Mundkapsel ist klein und zylindrisch, za. 0,024 Mm. lang und 0,008 breit; der Pharynx misst in der Laenge $0,25 \mathrm{Mm}$., in der Breite 0,012, der Oesopliagus ist 0,75 Mm. lang bei 0,055 Min. groesster Breite; der roehrenfoermige Darm zeigt im vordern Teile dieselbe Breite, wie der Oesophagus, und wird nach hinten zu sehr breit; der Anus liegt 0,07 Mm. vom Hinterende.

Die Hoden sind gut entwickelt und muenden in ein Vas deferens, dessen vorderer Teil stark erweitert ist; die Spicula messen 0,068 und 0,291 Mm. in der Laenge. Hinter dem Anus liegen jederseits 8 Dornen, davon drei in den Seitenlinien und drei ventral nahe der Medianlinie.

Fundort: Proventriculus der folgenden Voegel:

Fulica atra L., 1766.

Anas boscas L., 1766.

Gallus domesticus L., 1766.

Unser Material stammt von diesem letzten Wirt her. 
Habitat: Proventriculo das seguintes aves: Fulica atra L. , 1766.

Anas boscas L., 1766.

Gallus domesticus L. 1766.

O nosso material provem deste ultimo hospedeiro.

Vacilamos antes de identificar o parasiio que descrevemos ao $T$. fissispina, porem a coincidencia com as descrições existentes alias muito deficientes, a falta de material para comparação e a convivencia intima a que são muitas vezes obrigados os hospedadores desta especie nos levou a concluir deste inodo.

\section{Tetrameres cruzi n. sp.}

(Est. 17, fig. 3-4)

Femea: Comprimento $2 \mathrm{~mm}$.

Largura $1,5 \mathrm{~mm}$.

Corpo arredondado, de côr vermelha, com fortes estriações transversaes e 4 sulcos lonjitudinaes, correspondendo aos campos medianos e lateraes; muitas vezes o parasito sofre uma torsão do eixo lonjitudinal ; as extremidades se apresentam como prolongamentos conicos. O tubo dijestivo é constituido como na especie precedente; a capsula bucal mede 0,016 a $0,020 \mathrm{~mm}$. de comprimento por $0,008 \mathrm{~mm}$. de largura; o farinje tem um comprimento de $0,016 \mathrm{~mm}$. e o esofago $0,62 \mathrm{~mm}$. por $0,9 \mathrm{~mm}$. de largura ; o intestino é constituido por um grande saco, repleto de detritos negros, que se estreita para a extremidade posterior e termina por um canal muito fino ; o anus fica situado a 0,074 a 0,100 mm. da extremidade posterior.

O aparelho genital é muito desenvolvido, o utero se apresenta repleto de ovos em diversos estadios evolutivos; são elipsoides e medem 0,050 a $0,060 \mathrm{~mm}$. de comprimento por 0,024 a $0,028 \mathrm{~mm}$. de largura maxima; muitos tem embrião completamente desenvolvido; a vulva fica situada a mais ou menos $0,3 \mathrm{~mm}$. da extremidade posterior.

Macho: Comprimento 1,17 a $1,4 \mathrm{~mm}$.

Largura $0,086 \mathrm{~mm}$.

O corpo é filiforme, sem espinhos, de cor branca e com fortes estriações transversaes.
Ich zoegerte etwas, den beschriebenen Parasiten mit $T$. fissispina zu identifizieren, kam aber doch zu diesem Schlusse in Folge der Uebereinstimmungen mit den vorliegenden, allerdings sehr unvollkommenen Beschreibungen bei mangelndem Vergleichsmateriale und in Erwaegung des engen Zusammenlebens, zu dem die Wirte oft genoetigt sind.

\section{Tetrameres cruzi n. sp.}

(Taf. 17, Fig. 3 -4).

Weibchen: Laenge: $2 \mathrm{Mm}$.

Breite 1,5 Mm.

Koerper rundlich rotgefaerbt, stark quergestreift, mit vier Laengsfurchen, die den Seiten-und Mittellinien entsprechen, oft um die Laengsachse gedreht, die Koerperenden als konische Fortsaetze erscheinend. Digestionstrakt, wie bei der vorhergehenden Art, die Mundkapsel 0,016-0,02 Mm. lang und 0,008 Mm. breit; der Pharynx 0,16, der Oesophagus $0,62 \mathrm{Mm}$. lang; Darm aus einem grossen, mit schwarzem Detritus gefuellten Sacke bestehend, nach dem Hinterende zu verengert und in einen sehr feinen Kanal endend; Anus 0,074-0,1 Mm. vom Hinterende.

Genitalsystem sehr entwickelt; Uterus mit Eiern in verschiedenen Entwicklungstadien angefuellt, die eifoermig, 0,050-0,060 Mm. lang und 0,024-0,028 breit sind und deren viele einen voellig entwickelten Embryo er thalten; Vulva za. $0,3 \mathrm{Mm}$. von Hinterende.

Maennchen: Laenge: 1,17-1,4 Mm.

Breite: 0,085 Mm.

Koerper fadenfoermig, unbedornt, von weisser Farbe, stark quergestreift. Darmkanal aehnlich demjenigen der vorhergehenden Art; Mundkapsel 0,012 Mm. lang und 0,004 $-0,005 \mathrm{Mm}$. breit; Laenge des Pharynx 0,093 Mm. bei 0,009 Breite; Oesophagus 0,29 Mm. 
O tubo dijestivo é semelhante ao da especie precedente; a capsula bucal mede 0,012 min. de comprimento por 0,004 a $0,005 \mathrm{~mm}$. de largura; o farinje mede $0,093 \mathrm{~mm}$. de comprimento por $0,009 \mathrm{~mm}$. de largura; o comprimento do esofago é de $0,29 \mathrm{~mm}$. por 0,024 mm de largura; o anus fica situado a 0,132 $\mathrm{mm}$. da extremidade posterior.

O aparelho genital é constituido por grandes testiculos que ocupam a maior parte da cavidade do parasito e um volumoso canal deferente; os espiculos são de dimensões muito diferentes: o menor mede 0,082 e $o$ maior 0,651 a $0,787 \mathrm{~mm}$. de comprimento. Ha 4 pares de papilas, na extremidade posterior dos quaes um preanal e tres postanaes.

Habitat: Proventriculo de Bucro swainsoni GRAY, 1847 e Melanerpes flavifrons (VIEILL, i818).

Tipo no Instituto OSWALDO CRUZ.

Tetrameres minima $\mathrm{n}$. $\mathrm{sp}$.

(Est 19, fig. 8; est. 22-23).

Femea: Comprimento $0,78 \mathrm{~mm}$.

Largura : 0,64 $\mathrm{mm}$.

Corpo de cor vermelha, arredondado e com forte estriação transversal; as extremidades se apresentam como prolongamentos conicos, muito moveis e retrateis.

O tubo dijestivo é constituido como no T. fissispina; a capsula bucal mede 0,012 mm. de comprimento por 0,007 de largura; o faringe mede 0,073 de comprimento por 0,01 mm. de largura; o esofago tem um comprimento de $0.49 \mathrm{~mm}$. por $0,05 \mathrm{~mm}$. de largura maxima: o intestino é constituido por um saco que se extreita para traz formando um canal muito fino que termina no anus o qual acha-se situado a $0,068 \mathrm{~mm}$. da extre midade posteripr. O aparelho genital ocupa grande parte da cavidade do nematoideo; o utero se apresenta repleto de ovos, dos quaes muitos contem um embrião completamente desenvolvido; a vulva fica situada na extremidade posterior, acima do anus; os ovos medem cerca de $0,045 \mathrm{~mm}$. de comprimento por $0,024 \mathrm{~mm}$. de largura maxima. Macho: Comprimento $1,4 \mathrm{~mm}$. lang und 0,024 breit; Anus 0,132 Mm. von der Schwanzspitze entfernt. Der Genitalapp?rat besteht aus einem grossen Hoden, welche die Leibeshoehle des Wurmes zum groessten Teile ausiuellen und einem volumoesen Vas deferens; Spicula von sehr ungleichen Dimensionen, das kleinere 0,082, das groessere $0,651-0,787 \mathrm{Mm}$. lang. Es finden sich vier Papillenpaare am Hinterende, davon eines praeanal und drei postanal.

Fundort: Proventriculus von Bucco swaisoni GRAY, 1847 und Melanerpes flavifrons (VIEILL., 1818).

Typus im Instituto OSWALDO CRUZ.

Tetrameres minima $\mathrm{n}$. $\mathrm{sp}$. (Taf. 19, Fig. 8; Taî. 22-23).

Weibchen : Laenge : 0,78 Mm.

Breite : 0,64 Mm.

Koerper von roter Farbe und runder Form mit starker Querstreifung, seine beweglichen und retraktilen Enden konische Fortsaetze bildend. Verdauungstrakt, wie bei $T$. fissispina gebildet; Mundkapsel 0,012 Mm. lang, 0,007 breit; Pharynx in der Laenge 0,073 , in der Breite 0,01 Mm.; Oesophagus 0,49 Mm. lang, bei 0,05 groesster Breite; Darm aus einem Sack bestehend, der nach hinten zu duenner wird und mit einem feinen Kanale an dem $0,068 \mathrm{Mm}$. vor der Schwanzspitze gelegenen After endigt. Der Geschlechtsapparat nimmt den groessten Teil der Leibeshoehle ein; der Uterus zeigt sich mit Eiern gefuellt, von denen viele schon einen gut entwickelten Embryo einschliessen; sie sind za. $0,045 \mathrm{Mm}$. lang bei 0,024 groesster Breite.

Vulva am Hinterende vor dem Anus.

Maennchen: Laenge: 1,4 Mm. 
Devido ao pequeno tamanho e a grande transparencia os machos são deficeis de encontrar. Apenas pudemos examinar um exemplar, o qual não pode ser conservado, e de que damos figura.

.Corpo com lijeiras estriações transversaes, capsula bucal muito pequena, espiculos desigues sendo um muito grande com cerca de $0,99 \mathrm{~mm}$. e o outro pequeno com $0,1 \mathrm{~mm}$. de comprimento.

Habitat: Proventriculo de Tachyphonus cristatus bruneus (SPIX, 1824).

O parasito parece ser comum nestas aves, pois todos os exemplares que examinamos estavam parasitados.

Tipo no Instituto OSWALDO CRUZ.

Esta especie é muito proxima da precedente; com tudo se distingue com facilidade pelas dimensões inferiores e tamanho relativo dos espiculos.

Fevereiro de 1914.

Wegen ihrer geringen Groesse und grosser Durchsichtigkeit sind die Maennchen schwer zu finden. Ich habe nur das abgebildete Exemplar untersucht, welches nicht konserviert werden konnte. Koerper leicht quergestreift, Mundkapsel sehr klein, Spicula sehr ungleich, das eine sehr gross, 0,99 , das andere sebr kleine $0,1 \mathrm{Mm}$. lang.

Fundort: Proventriculus von Tachyphonus cristatus bruneus (SPIX, 1824).

Der Parasit scheint bei diesen Voegeln gemein zu sein, da ihn alle untersuchten Exemplare beherbergten.

Typus im Instituto OSWALDO CRUZ.

Diese Art steht der vorigen sehr nahe, unterscheidet sich aber leicht durch die geringeren Dimensionen und die Groessenverhaeltnisse der Spicula.

Februar 1914. 
BIBILIOGREAFIA.

\section{Bibliography.}

\section{CREPLIN}

DRASCHE

\section{LINSTOW}

V. LINSTOW

V. LINSTOW

V. LINSTOW

V. LINSTOW

RAILLIET

WIEGMANN

LEIDY

DUJARDIN

STILES \& HASSALL

WIEDMAN

SEURAT, L. G

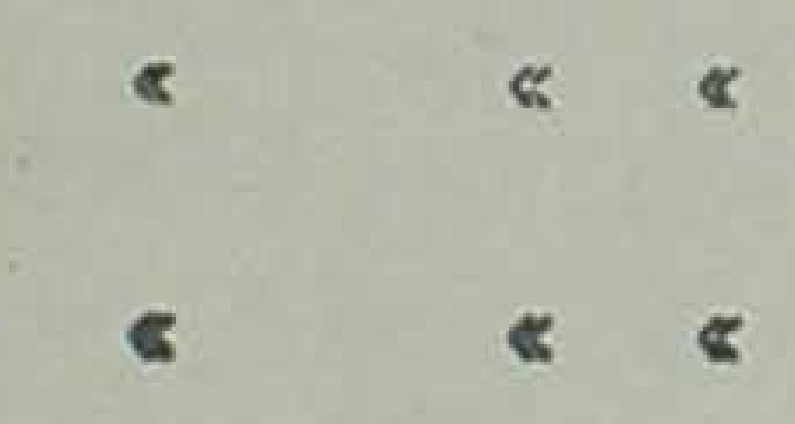

1846 Nachträge zu Gurlt's Verz eichniss der Thiere, bei welchen Entozoen gefunden warden sind. Arch. f. Naturg. V. 12 , p. 129.

1883 Revision der in der Nematoden Samunlung des k. k. Zoolog. Hofcabinetes de in dlichen Original-Exemplare Diesing's und Molin's. (Aus den Verhandlungen de k. k. Zoologische batanischen Gesellschaft in Wien. Wein. Pag. 10 (114).

18781889 Compendium der Helminthologie. Hannover.

1877 Helmintholojica. Arch. f. Naturg. V. 43, p. 1.

1876 Helminthologische Beobachtungen. Arch. f. Naturg. V 42, p. 1.

1879 Helminthologische Studien. Arch. f. Naturg. V. 45, p. 165.

1899 Zur Kenntiniss der Genera Hystrichis und Tropidocerca. Arch. f. Naturg. V. 65. p. 155.

1895 Traite de Zoologie Medicale et Agricole. Pag. 544-545.

1835 Bericht ueber die Fartschritte der Zoologie im Jahre 1834. (Entozoen) Arch. f. Naturg. V. 1, pp. 337-338.

1887 Notices of Nematoid Worms. Proc. of the Acad. of Natural Sciences of Philadelphia. Pag. 308.

1845 Hist. Natur. des Helmithes. Pag. 293.

1905 The determintion of generc types and a list of roundworm genera, with their original and type species. U. S. Department of Agriculture. Bureau of animal Industry Bull. N. 79.

1913 A study of metazoan parasites faund in the Philadelphia Zoological Garden. Proc. of the Acad. of Nat. Sciences of Philadelphia. V. LXV, p. 126.

1914 Sur la morphologie de lovoejecteur des Tropidocerca. Campt. Rend. Soc. Biol. V. 76, p. 173, Fig. 1-3.

1914 Sur un Tropidocerca parasite d'un Echassier. Comp. red. Soc. Biol. V. 76 , p. 778 , fig. $1-8$.

1914 Sur em Nématode parasite de flammant rose. Compt. rend. Soc. Biol. V. 76, p, 814, fig, 1-4. 


\section{Explicação das figuras.}

Est. 16, fig. 1.-Tetrameres fissispina-femea muito nova.

Est. 17, fig. 2.-Tetrameres fissispinafemea adulta.

Est. " fig. 3.-Tetrameres cruzi-macho. Est. " fig. 4.-Tetrameres cruzi-femea. Est. 18, fig. 5. - Tetrameres paradoxacauda de macho (segundo DRASCHE).

Est. « fig. 6.-Tetrameres fissispinacauda de macho.

Est. " fig. 7. - Tetrameres fissispina-tubo dijestivo de macho.

Est. 19, fig. 8. - Tetrameres minima-macho.

Est. * fig. 9.-Tetramure; fissispinacorte lonjitudinal de femea aduita.

Est. " fig. 10.-Tetrameres fissispina-femea em corte de proventiculo de galinha.

Est. 20, fig. 11. - Tetrameres fissispina-femea em corte de proventrlculo de galinha (no centro do intestino ve-se a abertura do esofago cerca das grandes celulas glandulares aí existentes).

Est. 20, fig. 12. - Tetrameres fissispinacorte transversal de femea mostrando as celulas glandulares piriformes que guarnecem a abertura posterior do esofago.

Est. " fig. 13. - Tetiameres fissispina-femea; corte lonjitudinal passando ao nivel da abertura do esofago no intestino.

Est. 21, fig. 14. - Tetrameres fissispinaabertura do esofago no intestino (fig. 13 ampliada).

Est. " fig. 15. - Tetrameres fissispina-femea; corte transversal na metade posterior.

Est. " fig. 16. - Tetrameres fissispina-femea; corte transversal passando perto $\mathrm{d} z$ extremidade posterior.

Est. 22, fig. 17. - Tetrameres minima-femea em corte de proventriculo de $T$. cristatus bruneus.

Est. « fig. 18. - Tetrameres minima-femea; idem, idem.

Est. " fig. 19. - Tetrameres minima-femea; idem, idem.

Est. 23, fig. 20.--Tetrameres minima-macho em corte de proventriculo de $T$. cristatus bruneus (o parasito está alojado em uma glandula).

Est. * fig. 51.-Tetrameres minima-corte de proventriculo de $T$. cristatus bruneus (o parasito está na cavidade do orgão.

\section{Erklaerung der Abbildungen.}

Taf. 16, fig. 1. - Tetrameres fissispina-Sehr junges Weibchen.

Taf. 17, fig. 2. - Tetrameres fissispina-Erwachsenes Weibchen.

Taf. 17 fig. 3-Tetrameres cruzi-Maennchen.

Taf. 17 fig. 4. - Tetrameres cruzi-Weibchen.

Taf. 18, fig. 5- Tetrameres paradoxa-Schwanz des Maennchens (nach DRASCHE).

Taf. 18 fig. 6. - Tetrameres fissispina-Schwanz des Maennchens.

Taf. 18 fig. 7.-Tetrameres fissispina-Tractus digestivus des Maennchens.

Taf. 19, fig. 8.-Tetrameres mınima-Maennchen.

Taf. 19 fig. 9. - Tetrameres fissispina-Laengsschnitt eines erwachsenen Weibchens.

Taf. 19f ig. 10. - Tetramercs fissispina-Weibchen-Schnitt eines Huehner-Vormagens

Taf. 20, fig. 11. - Tetrameres fissispına-Weibchen-Schnitt eines Huehner-Vormagens (in der Mitte des Darms sieht man die Oesophagusoeffnung umgeben von den sich dort befindenden grossen Druesenzellen).

Taf. 20 fig. 12. - Tetrameres fissispina-Querschnitt eines Weibchens. Man sieht die birnfoermigen Druesenzellen,welche die hintere Oesophagusmuendung umgeben.

Taf. 20 fig. 13 - Tetrameres fissispina-Weibchen; Laengsschnitt auf der Hoehe der Oesophagusoeffnung im Darm.

Taf. 21, fig. 14. - Tetrameres fissispina-Oesophagusoeffnung im Darm (Vergroesserung der Figur 13.1

Taf. 21 fig. 15. - Tetrameres fissispina-Weibchen; Querschnitt in der hinteren Haelfte.

Taf. 21 fig. 16. - Tetrameres fissispina-Weibchen; Querschnitt in der Naehe des Hinterendes.

Taf. 22, fig. 17. - Tetrameres minima-Weibchen; Schnitt des Vormagens eines T. cristatus bruneus

Taf. 22 fig. 18. - Tetrameres minima-Weibchen ; idem, idem.

Taf. 22 fig. 19.-Tetrameres minima-Weibchen; idem, idem.

Taf. 23, fig. 20.- Tetrameres minima-Maennhen; Schnitt des Vormagens eines $T$. cristatus bruneus (der Parasit befindet sich in einer Druese).

Taf. 23 fig. 21.-Tetrameres minima-Schnitt des Vormagens eines $T$. cristatus bruneus (Der Parasit befindet sich in der Organhoehle). 


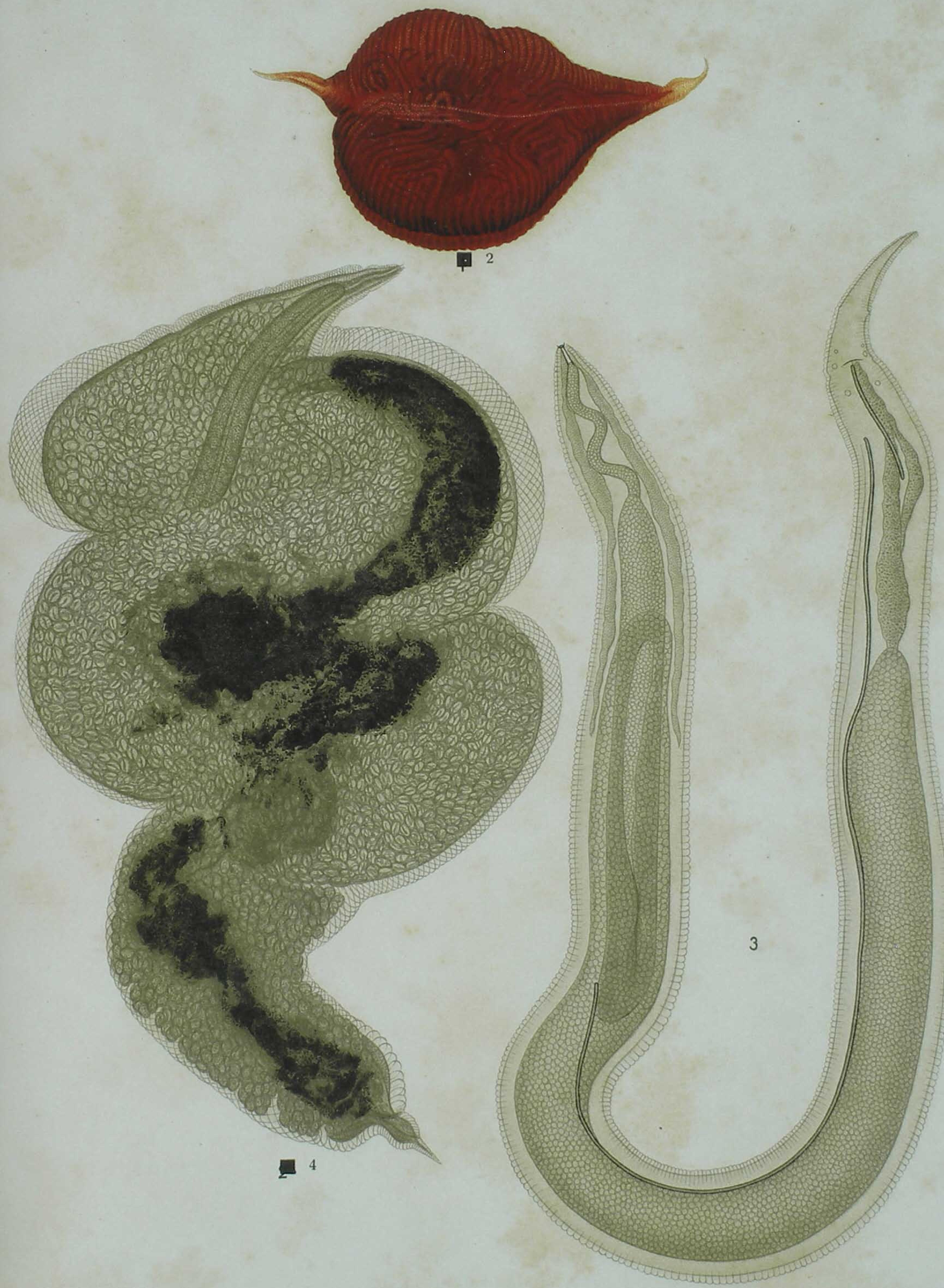

RUD. FISCHER, del 

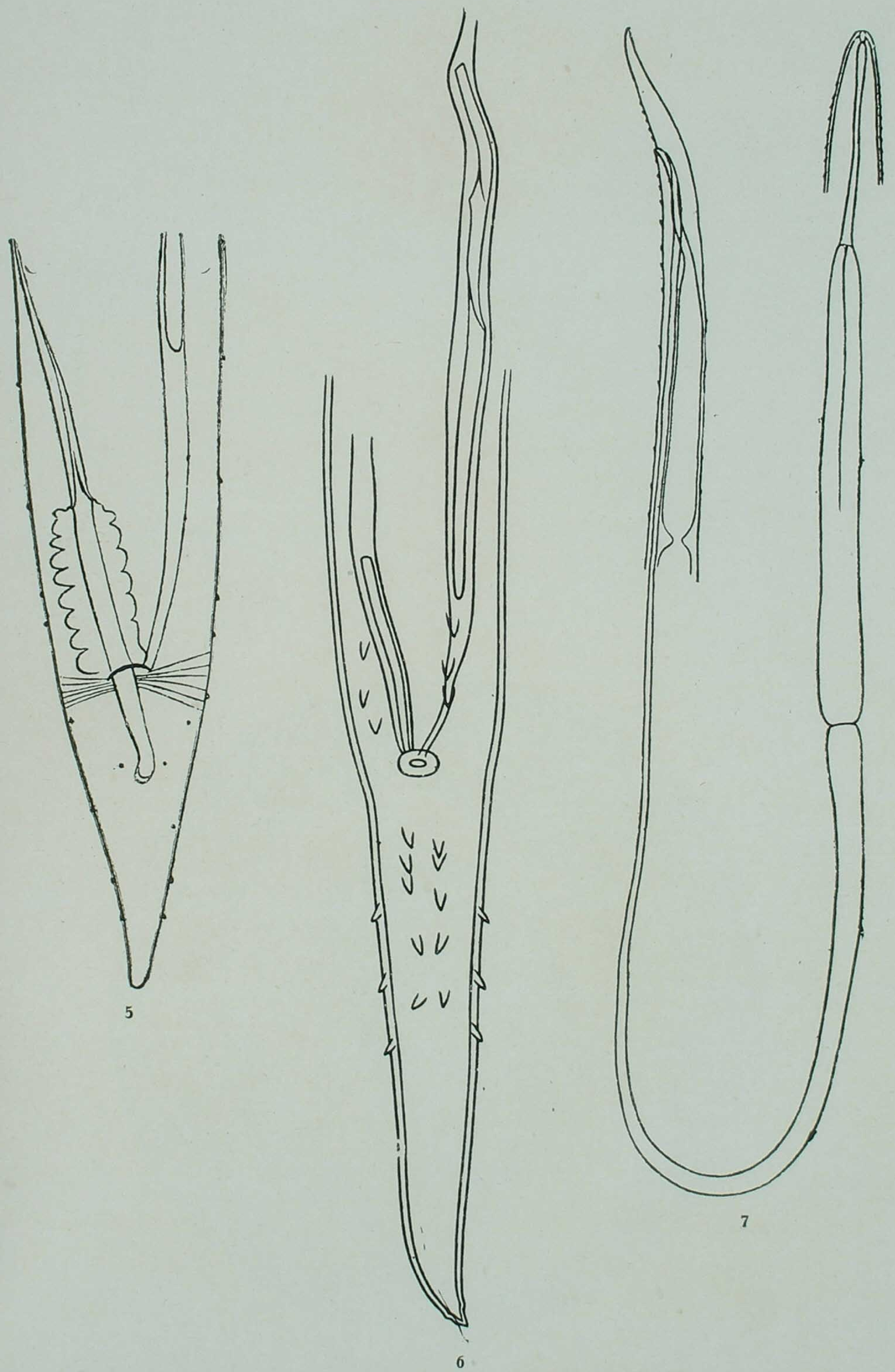

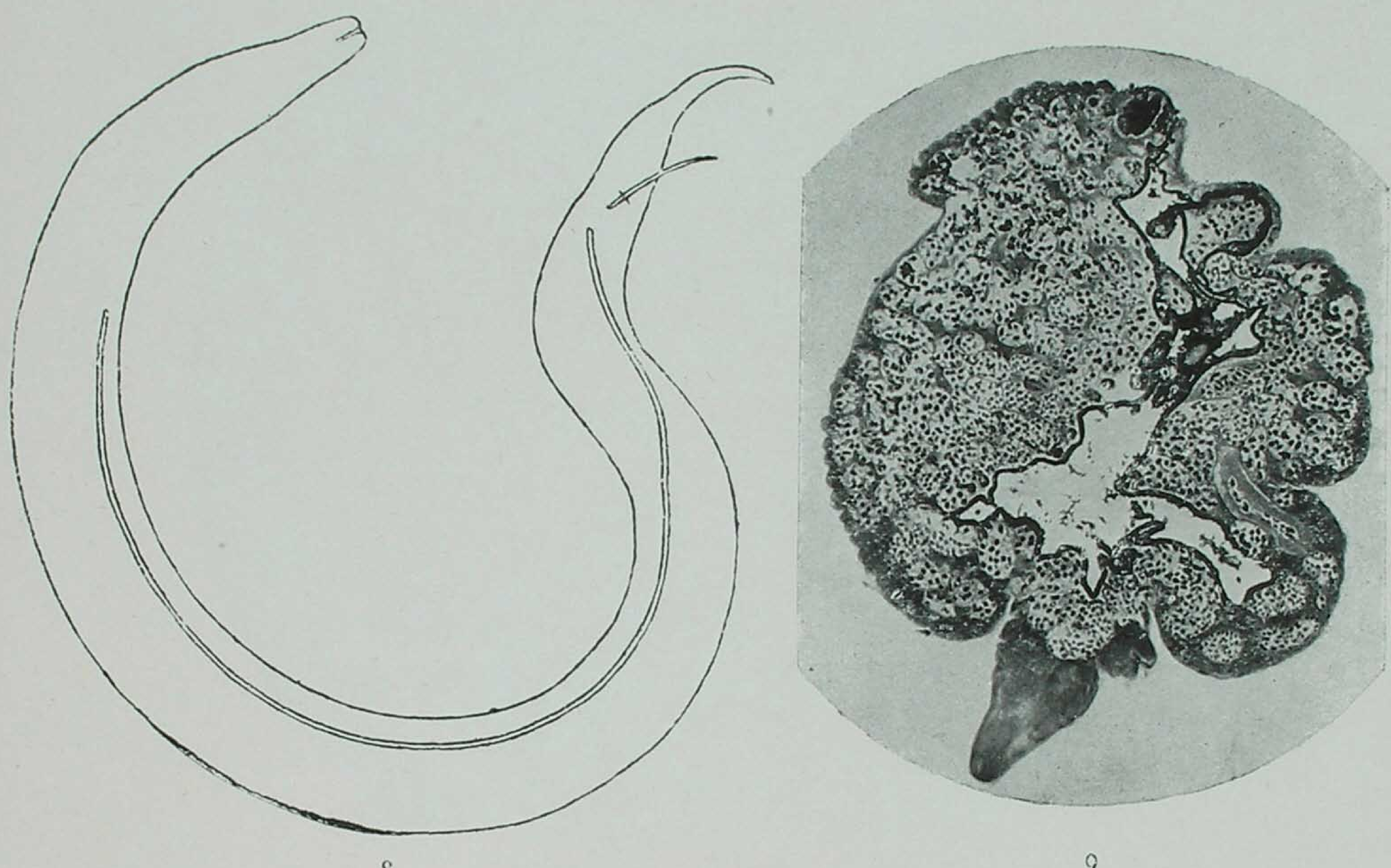

8

9

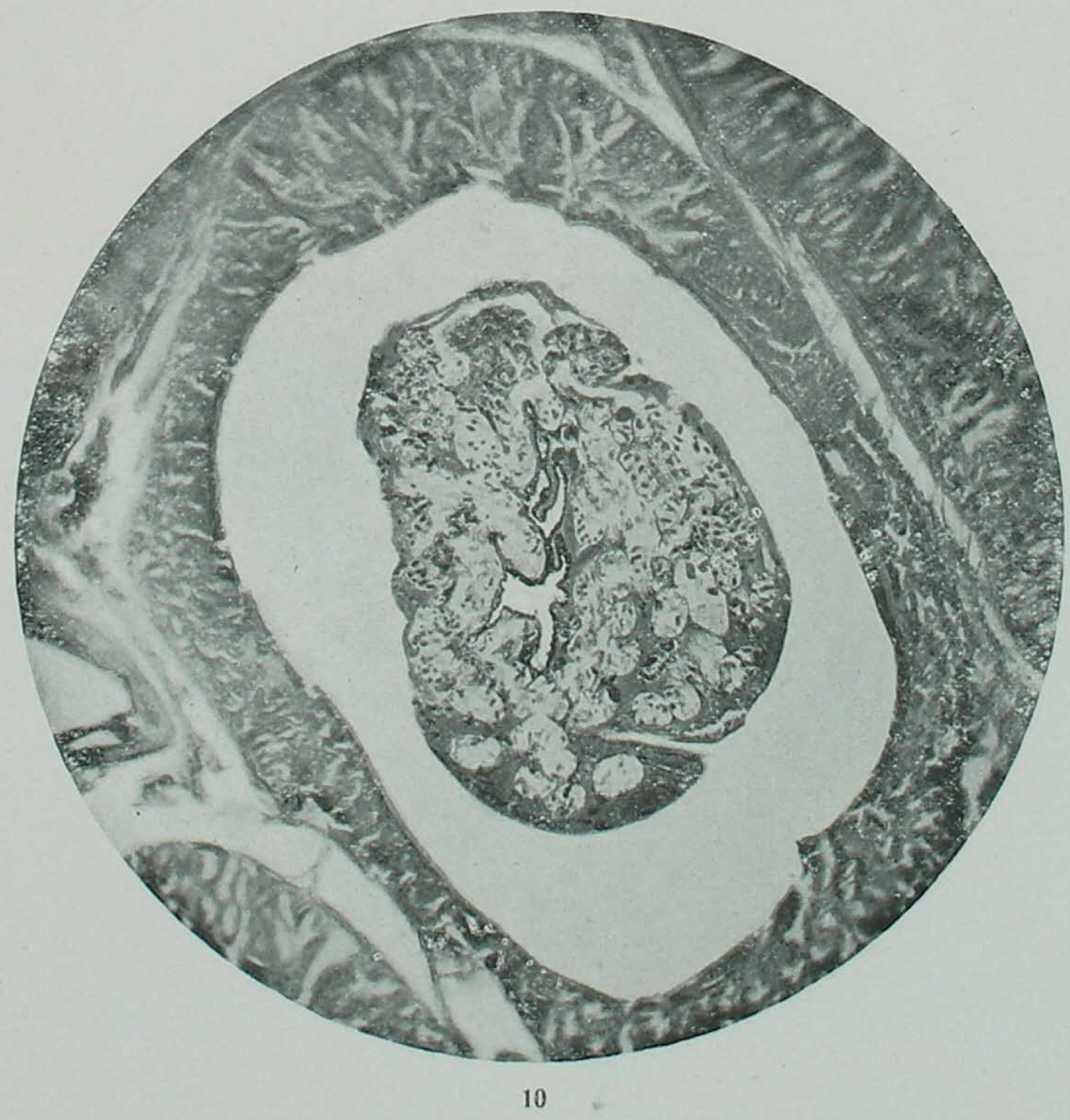




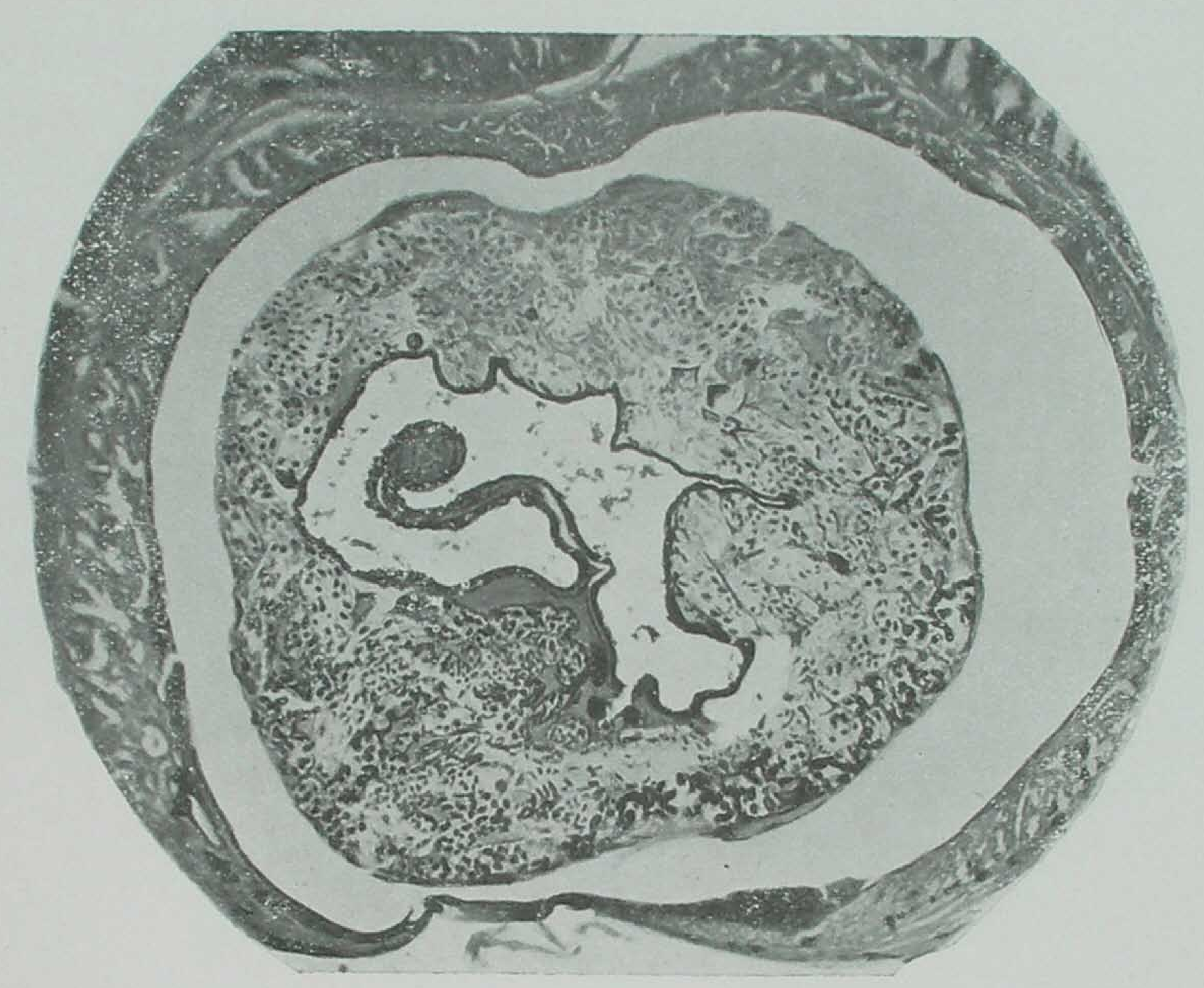

11
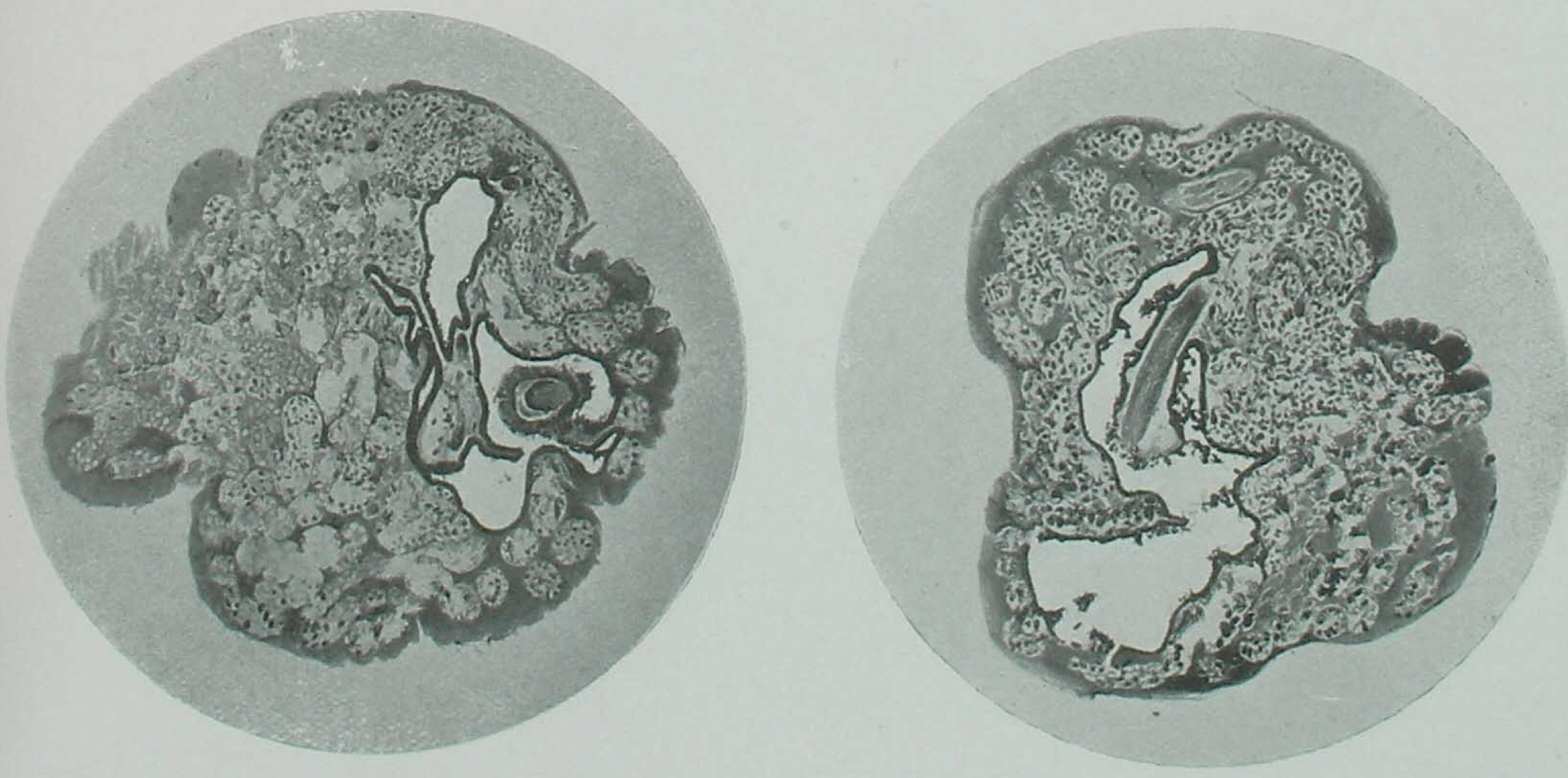

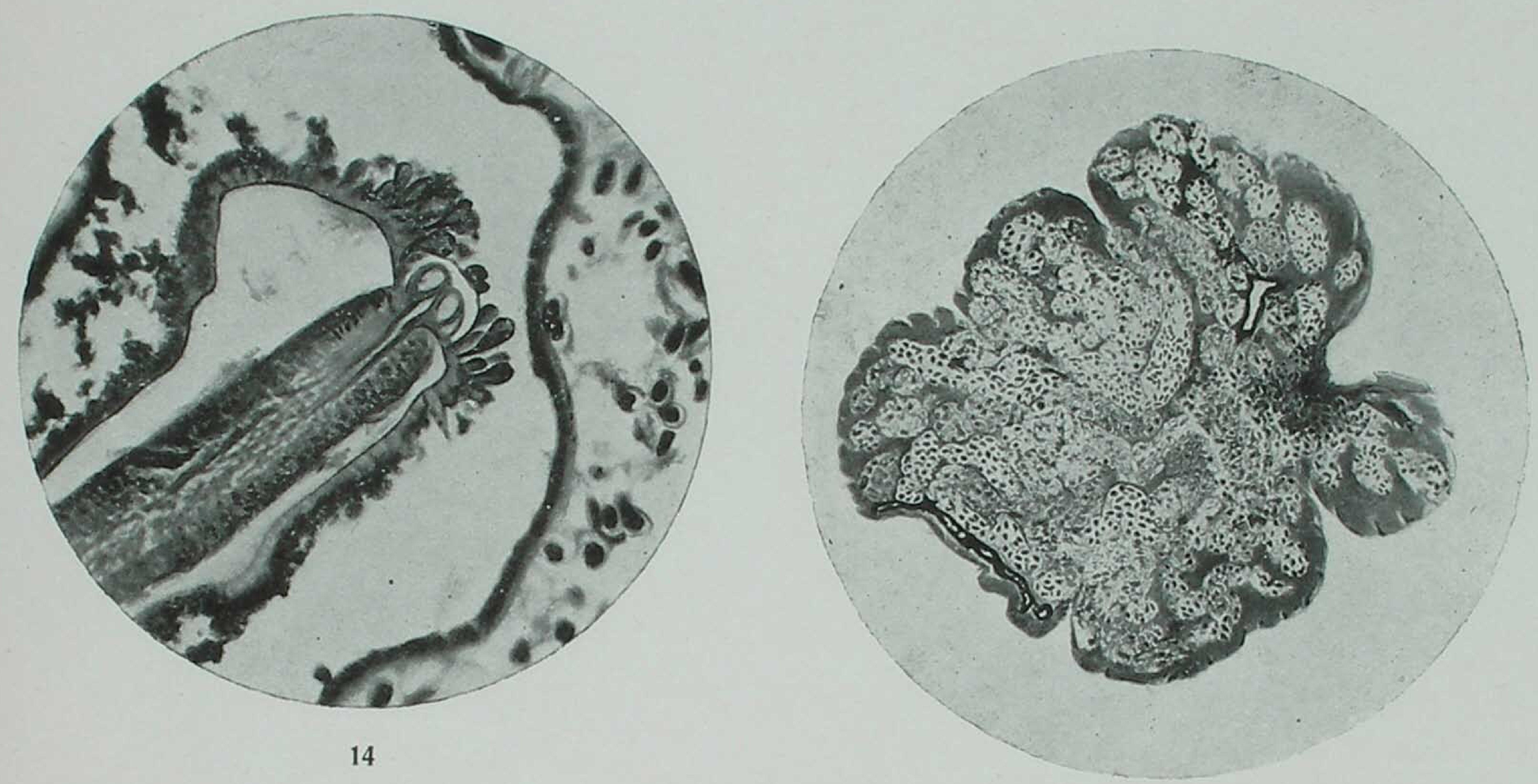

15

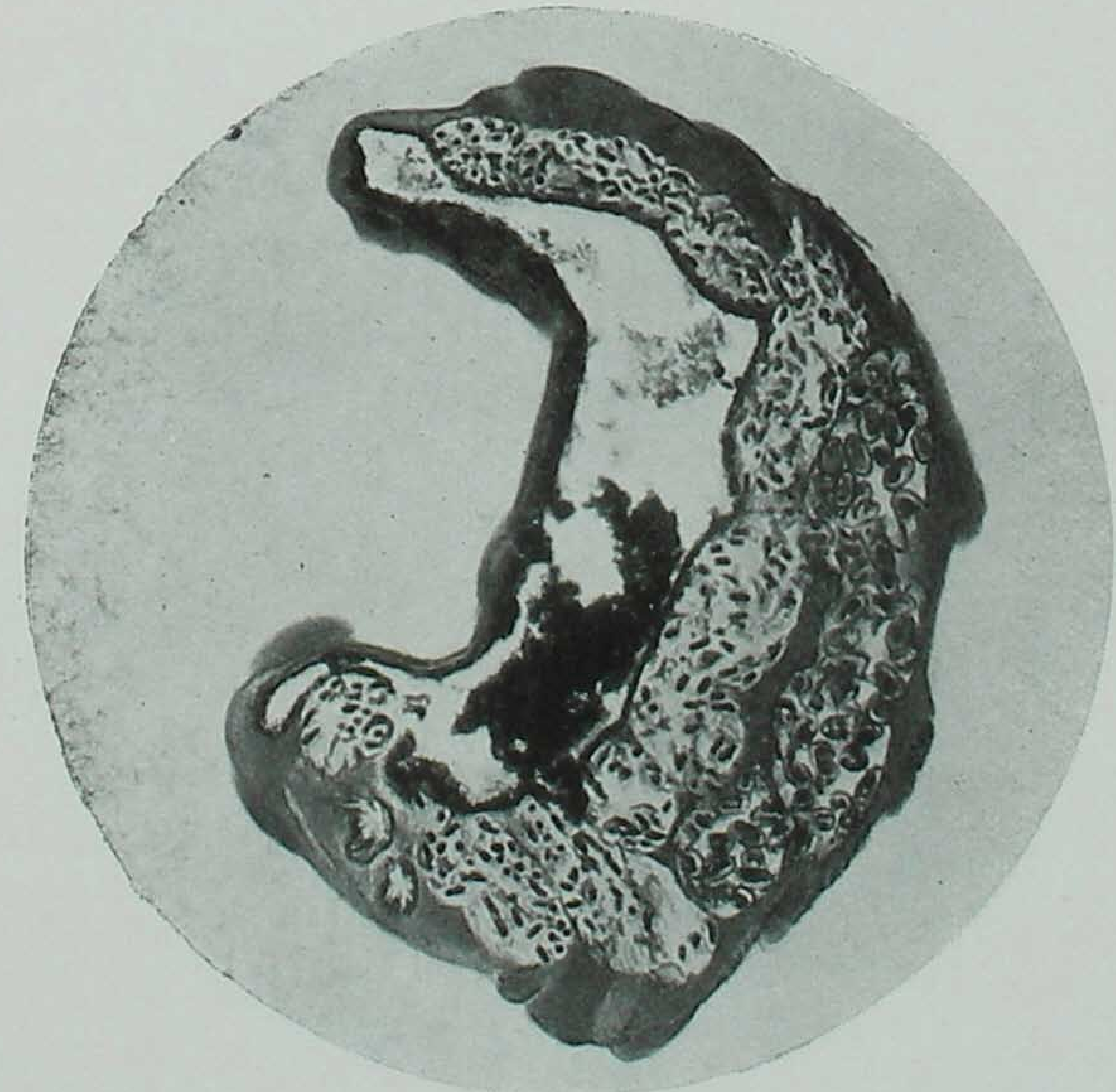




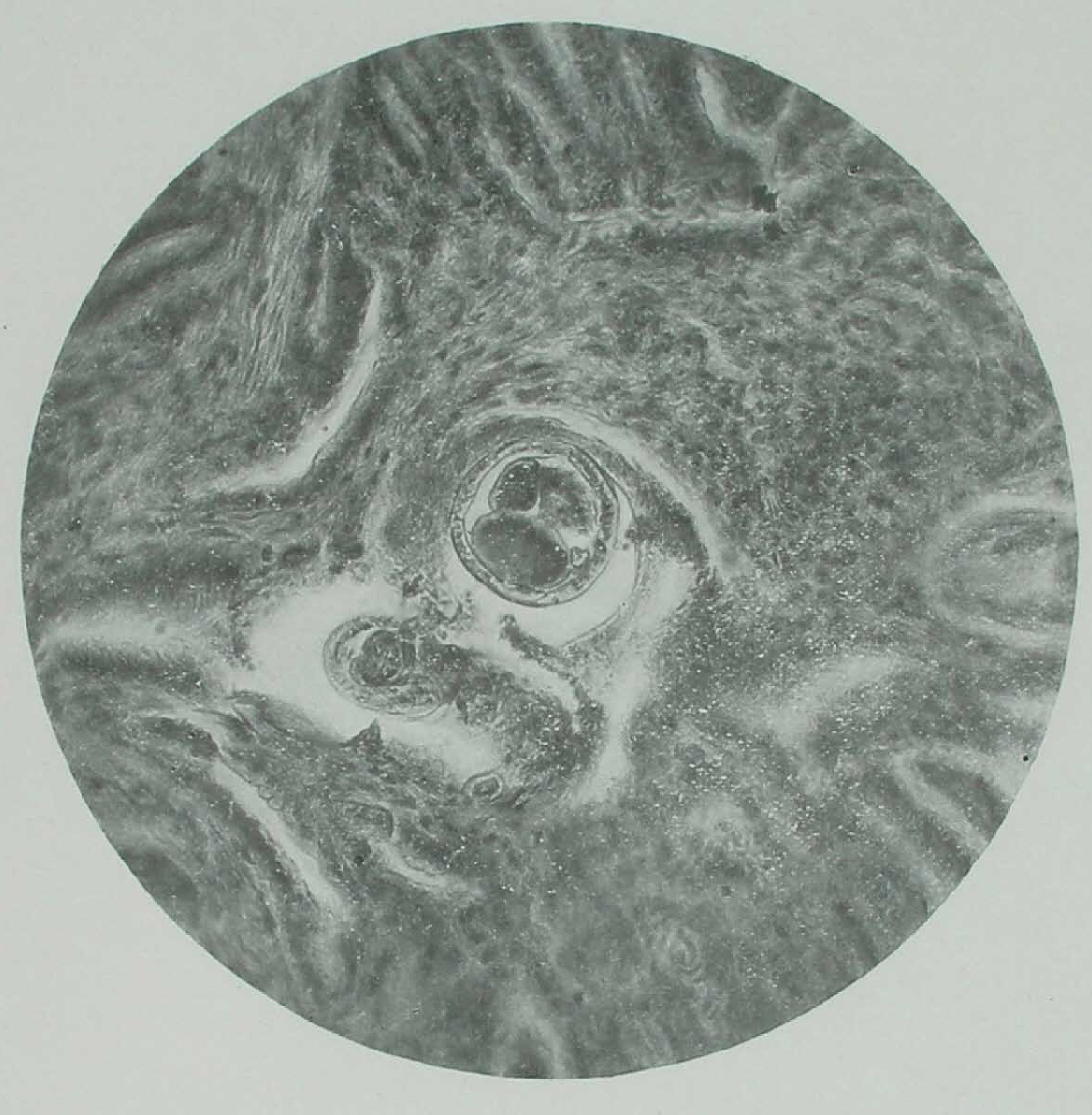

20

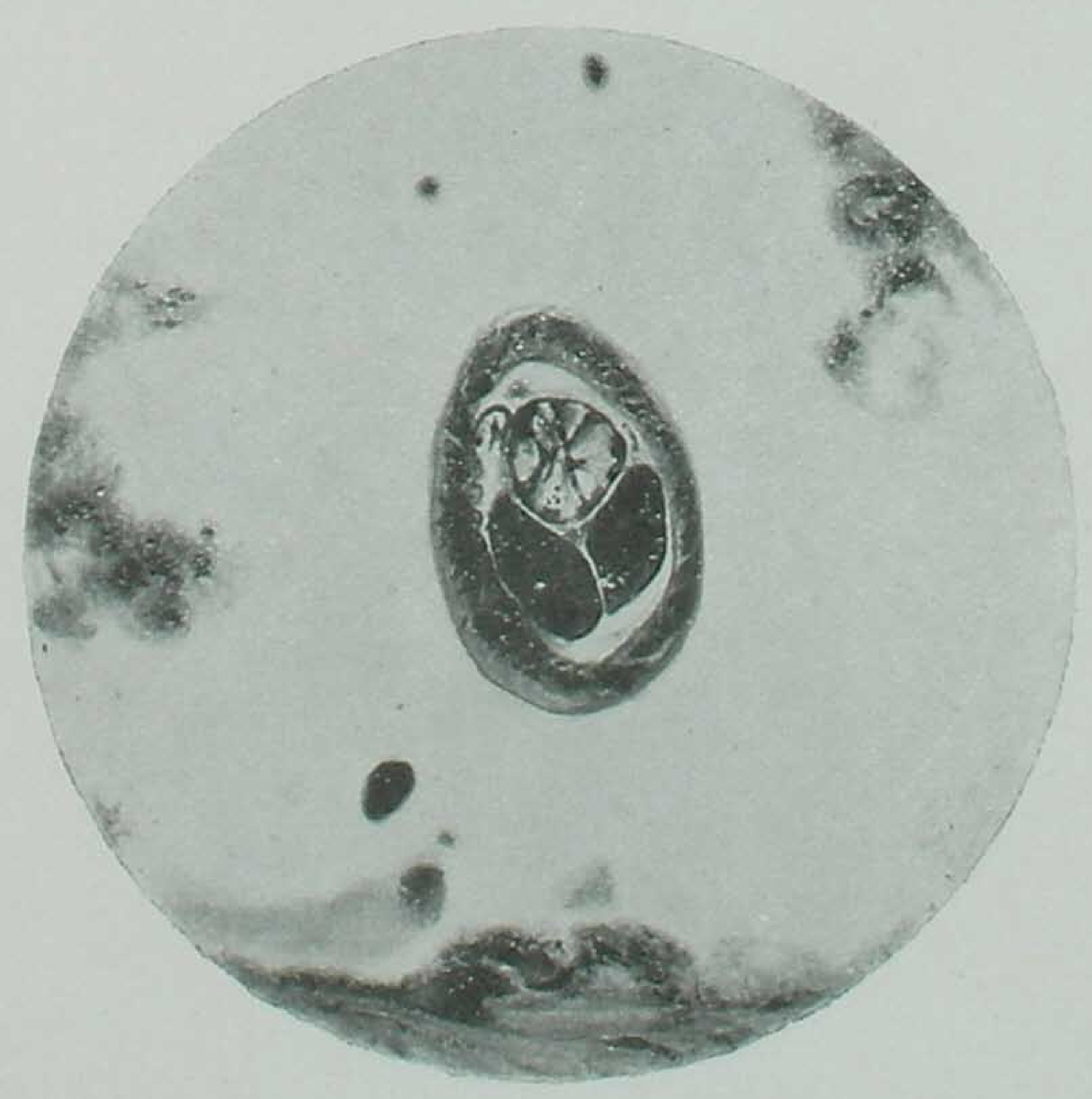

